Research Article

\title{
Disease Mutation Study Identifies Critical Residues for Phosphatidylserine Flippase ATP11A
}

\author{
Kuanxiang Sun, ${ }^{1}$ Wanli Tian, ${ }^{1}$ Xiao Li, ${ }^{1}$ Wenjing Liu, ${ }^{1}$ Yeming Yang ${ }^{(D},{ }^{1}$ \\ and Xianjun Zhu $\mathbb{C}^{1,2}$ \\ ${ }^{1}$ Department of Laboratory Medicine, Sichuan Provincial Key Laboratory for Human Disease Gene Study, Sichuan Provincial \\ People's Hospital, School of Medicine, University of Electronic Science and Technology of China, Chengdu, Sichuan, China 610072 \\ ${ }^{2}$ Department of Ophthalmology, First People's Hospital of Shangqiu, Shangqiu, Henan, China 476100
}

Correspondence should be addressed to Yeming Yang; nickyym@126.com and Xianjun Zhu; xjzhu@uestc.edu.cn

Received 9 February 2020; Accepted 21 April 2020; Published 2 June 2020

Academic Editor: Graziano Pesole

Copyright ( 2020 Kuanxiang Sun et al. This is an open access article distributed under the Creative Commons Attribution License, which permits unrestricted use, distribution, and reproduction in any medium, provided the original work is properly cited.

\begin{abstract}
Phosphatidylserine flippase (P4-ATPase) transports PS from the outer to the inner leaflet of the lipid bilayer in the membrane to maintain PS asymmetry, which is important for biological activities of the cell. ATP11A is expressed in multiple tissues and plays a role in myotube formation. However, the detailed cellular function of ATP11A remains elusive. Mutation analysis revealed that I91, L308, and E897 residues in ATP8A2 are important for flippase activity. In order to investigate the roles of these corresponding amino acid residues in ATP11A protein, we assessed the expression and cellular localization of the respective ATP11A mutant proteins. ATP11A mainly localizes to the Golgi and plasma membrane when coexpressed with the $\beta$-subunit of the complex TMEM30A. Y300F mutation causes reduced ATP11A expression, and Y300F and D913K mutations affect correct localization of the Golgi and plasma membrane. In addition, Y300F and D913K mutations also affect PS flippase activity. Our data provides insight into important residues of ATP11A.
\end{abstract}

\section{Introduction}

Phospholipids of eukaryotic cell membranes are asymmetrically distributed, with phosphatidylserine (PS) and phosphatidylethanolamine (PE) concentrated mainly in the cytoplasmic leaflet of the membrane bilayer, while phosphatidylcholine (PC) and sphingolipids mainly positioned only on the exoplasmic leaflet [1-6]. Numerous physiological and biochemical processes, such as membrane stability and dynamics, cell polarity and migration, protein trafficking, cell apoptosis, cell signaling, platelet activation, neurodevelopment, blood coagulation, and sperm cell capacitation, are relied on the asymmetric distribution of phospholipids [2, 7-16]. Mounting evidence reveals that the asymmetrically distributed phospholipids are maintained by P4-ATPases (also called flippases), which translocate phospholipids from the exoplasmic leaflet to the cytoplasmic leaflet $[1,3-6,17]$. In mammals, 14 members of P4-ATPases, designated ATP8A1 through ATP11C, have been identified [18].
ATP8A1, ATP8A2, ATP8B1, ATP8B2, ATP8B4, ATP10A, ATP10D, ATP11A, and ATP11C are localized to the plasma membrane, whereas ATP9A, ATP9B, ATP10B, and ATP11B are localized to intracellular membranes $[1,4,7,15,19,20]$. Among the cell surface-localized P4-ATPases, ATP8A1, ATP8A2, ATP8B1, ATP11A, and ATP11C have been shown to catalyze the inward translocation of PS at the plasma membrane $[4,7,15,19,20]$. P4-ATPases, similar to other Ptype ATPases such as $\mathrm{Na}^{+}$and $\mathrm{K}^{+}$-ATPase, are depending on heterodimeric interaction with a $\beta$-subunit CDC50 to proper folding and trafficking [21]. In the mammalian genome, there are three isoforms of CDC50 family: $C D C 50 A, C D C 50 B$, and CDC50C (also named TMEM30A, TMEM30B, and TMEM30C, respectively). TMEM30A interacts with the vast majority of $\mathrm{P} 4$ ATPases, including ATP11A, and is critical for its proper functions [21].

Thus far, the physiological functions of the majority of mammalian P4-ATPases were still unclear. Only mutations of several P4-ATPases were reported to cause severe human 
disease [22]. For instance, mutations identified in ATP8B1 are associated with liver disorders such as progressive familial intrahepatic cholestasis and hearing loss [23, 24]. Mutations in ATP8A2 cause severe neurological disorders such as cerebellar ataxia, mental retardation, and disequilibrium syndrome $[25,26]$. ATP11C plays a crucial role in the differentiation of B cell in lymphopenia, anemia, and intrahepatic cholestasis in mice [27-30]. ATP11A exhibits largely similar cellular distribution with ATP8A1 and ATP8A2 [19, 31]. However, only a few studies were reported on ATP11A. ATP11A is ubiquitously expressed in various tissues [19] and the deletion of Atp11a results in lethality during embryogenesis. Recent research indicated that the phospholipid flippase complex of ATP11A and CDC50A acts as a molecular switch for PIEZO1 activation that governs proper morphogenesis during myotube formation [32]. The detailed cellular function of ATP11A remains to be determined.

A previous study has reported ATP8B1 mutations L127P and $\mathrm{E} 981 \mathrm{~K}$ in patients with familial intrahepatic cholestasis type 1 (PFIC1), while the mutation $\mathrm{I} 344 \mathrm{~F}$ is identified in patients with benign recurrent intrahepatic cholestasis type 1 (BRIC1) [33, 34]. An ATP8B1 homozygous mutation L127V causes intrahepatic cholestasis in two Omani siblings [35]. The equivalent mutations of bovine ATP8A2 are I91P, $\mathrm{I} 91 \mathrm{~V}, \mathrm{~L} 308 \mathrm{~F}$, and E897K. To elucidate the functional consequences of flippase disease mutations, Gantzel et al. investigated the effect of mutations of those residues on expression and activity of ATP8A2 and found out the essential roles of these residues to the flippase translocation process [36]. Mutations of the I91 and L308 residues in ATP8A2 are positioned near proposed translocation routes in the protein. Mutation of the E897 residue is located at the exoplasmic loop between transmembrane helix 5 and 6. This mutational analysis suggested that 191, L308, and E897 residues are important for the transport of phospholipids.

In order to investigate the importance of these abovementioned corresponding amino acid residues of ATP11A in the transporting phospholipids process, we matched these mutations to the equivalent sites of human ATP11A, which are $\mathrm{I} 80 \mathrm{P}, \mathrm{I} 80 \mathrm{~V}, \mathrm{Y} 300 \mathrm{~F}$, and $\mathrm{D} 913 \mathrm{~K}$, respectively, and introduced those mutations into ATP11A ORF by site-direct mutagenesis technique. By investigating the expression pattern and flippase activity of these mutated ATP11A proteins, we demonstrated that variants of Y300F and D913K affected correct Golgi localization and the amount of PS internalization in the plasma membrane. Additionally, Y300F mutation led to a decrease in ATP11A expression. This data provides insight into residues important for expression and cellular localization of ATP11A protein.

\section{Materials and Methods}

2.1. Cell Culture and Transfections. HEK 293T and COS7 cells (American Type Culture Collection (ATCC, Manassas, VA, USA) were cultured in $4 \mathrm{mM} \mathrm{L}$-glutamine and $4500 \mathrm{mg} / \mathrm{ml}$ glucose DMEM (HyClone) supplemented with $10 \%$ fetal calf serum (Gibco) and $1 \%$ antibiotics (HyClone) at $37^{\circ} \mathrm{C}$ in $5 \%$ CO2 atmosphere. When HEK $293 \mathrm{~T}$ cell density is $50 \%$ on $6-$ well plates and Cos7 cell density is $30 \%, 1 \mu \mathrm{g}$ plasmid DNA was added with Lipofectamine 3000 (Invitrogen), $50 \mu \mathrm{l} / \mathrm{ml}$ OPTI-MEM (Gibco)/DMEM. Cells were harvested after 3648 hours.

2.2. Site-Directed Mutagenesis. Primers carrying the mutations were designed using NEBaseChanger software. Primer sequences: I80P-F: 5 '-TTTCCTTATCCCATTTCTGGTGC AG-3', I80P-R: $5^{\prime}$-TAAAAGTTGGCTACTCTTC-3'; I80VF: $5^{\prime}$-TTTCCTTATCGTATTTCTGGTG-3', I80V-R: $5^{\prime}$ TAAAAGTTGGCTACTCTTC-3'; Y300F-F: $5^{\prime}$-CTCATTG TGTTCCTCTGCATTCTG-3', Y300F-R: $5^{\prime}$-GAACGCATT CATCGATTTTTC-3'; D913K-F: $5^{\prime}$-GACTTTGTACAAG ACCGCGTATC-3', D913K-R: 5' -TGTTGTGAAAACCCA CAG-3'. Mutations were introduced into the ATP11A ORF (NM_015205.2), which was cloned into the PCMV6 expression vector, using the $\mathrm{Q} 5{ }^{\circledR}$ Sit-direct mutagenesis kit (New England Biolabs, Ipswich, MA, USA) following the manufacturer's instruction. The mutated plasmids were sequenced to verify the successful introduction of the mutation.

2.3. Immunoblotting. 36-48 hours after transfection, HEK $293 \mathrm{~T}$ cells were lysed in RIPA lysis buffer $(150 \mathrm{mM} \mathrm{NaCl}$, $50 \mathrm{mM}$ Tris- $\mathrm{HCl}, 1 \%$ Triton X-100, 0.5\% sodium deoxycholate, $0.1 \%$ SDS, pH 7.4) supplemented with Complete Protease Inhibitor Cocktail (Roche). The protein concentration of the lysates was determined using a DC Protein Assay (Bio-Rad). Equal amounts of protein (20ug) were loaded onto a $10 \%$ polyacrylamide gel and $0.45 \mu \mathrm{M}$ nitrocellulose membrane (Millipore, Billerica, MA, USA, catalog \# HATF00010) was used for electrophoretic transfer. The blots were blocked with $8 \%$ nonfat dry milk in Tris-buffer saline with $0.1 \%$ Triton X-100 (TBST) for 2 hours at room temperature. Then, the membranes were incubated with the primary antibodies in blocking solution overnight at $4^{\circ} \mathrm{C}$. The following primary antibodies were used for the Western blotting: mouse antibodies against Flag (Sigma-Aldrich, St. Louis, MO, USA, dilution 1:5000) and mouse antibody against $\beta$-actin (Proteintech Group, Chicago, IL, USA, dilution $1: 2000)$. After the primary antibodies were removed, the membranes were washed three times with TBST for 15 minutes each time. The membranes were then incubated with anti-mouse HRP-conjugated secondary antibodies (1:5000; Bio-Rad, Hercules, CA, USA) for 2 hours at room temperature, and the signal was developed using Supersignal West Pico Chemiluminescent Substrate (Thermo Scientific). The relative intensity of the immunoreactive bands was quantified using the gel analysis tool provided in the ImageJ software. Normalization of the proteins of interest was performed relative to $\beta$-actin.

2.4. Immunocytochemistry. 36-48 hours after COS7 cells transfection, the transfected cells were washed twice with PBS, fixed with $4 \%$ paraformaldehyde, and washed three times with PBS. Cells were then permeabilized and blocked with normal goat serum, Triton $\mathrm{X}-100$, and $\mathrm{NaN}_{3}$ in PBS for 1 hour at room temperature. ATP11A protein was labeled with mouse anti-Flag antibody ( $1: 2000$, Sigma, Germany), and the endoplasmic reticulum (ER) was labeled with a rabbit anti-Calnexin antibody $(1: 1000$, Cell Signaling Technology, 


\begin{tabular}{|c|c|c|c|c|c|c|c|}
\hline ATP8A1 & I85 & L301 & E889 & ATP8A2 & I91 & L308 & E897 \\
\hline H. sapiens & SFFLFI ALLQ & VQI LI LFCI L & GQI LFERWCI & H. sapiens & AFFLFI ALLQ & VQI LVLFGI L & GQI LFERWCI \\
\hline P. troglodytes & SF F LF I ALLQ & VQI LI LFCI L & GQI LFERWCI & P. troglodytes & AFFLFI ALLQ & VQI LVLFGI L & GQI LFERWCI \\
\hline M. mulatta & SF F LFI ALLQ & VQI LI LFCI L & GQI LFERWCI & M. mulatta & AFFLFI ALLQ & VQI LVLFGI L & GQI LFERWCI \\
\hline C. lupus & SF F LF I ALLQ & VQI LI LFCI L & GQI LFERWCI & C. lupus & AF F LFI ALLQ & VQI LVLFGI L & GQI LFERWCI \\
\hline B. taurus & SFFLFI ALLQ & VQI LI LFCI L & GQI LFERWCI & B. taurus & AF FLF I ALLQ & VQI LVLFGI L & GQI LFERWCI \\
\hline M. musculus & SF F LF I ALLQ & VQI LI LFCI L & GQI LFERWCI & M. musculus & AF F LF I ALLQ & VQI LVLFGI L & GQI LFERWCI \\
\hline R. norvegicus & SFFLFI ALLQ & VQI LI LFCI L & GQI LFERWCI & R. norvegicus & AFFLFI ALLQ & VQI LVLFGI L & GQI LFERWCI \\
\hline G. gallus & AF F LFI ALLQ & I QI LI LFCI L & GQI LFERWCI & G. gallus & AFFLFI ALLQ & MQI LVLFGI L & GQI LFERWCI \\
\hline C. elegans & I FFLAI ALLQ & YRI I FLFFVL & GQTI FERW I & D. rerio & AFFLFI ALLQ & MQI LVLFGI L & GQI LFERWCI \\
\hline X. tropicalis & AF F LFI ALLQ & I QI I FLFGTL & GQI LFERWCI & X. tropicalis & AFF LFI ALLQ & VQI LVLFGI L & GQI LFERWCI \\
\hline ATP11A & I80 & Y300 & D913 & ATP11C & I78 & Y298 & D907 \\
\hline H. sapiens & FYFLI I FLVQ & AF LLVYLCI L & QQTLY DTAYL & H. sapiens & FYFLI I FL VQ & AF L I VYLF I L & QQP LY DAAYL \\
\hline P. troglodytes & FYFLI I FLVQ & AF LLVYLCI L & QQTLY DTAYL & P. troglodytes & FYFLI I FL VQ & AF LI VYLF I L & QQP LY DAAYL \\
\hline M. mulatta & FYFLI I FLVQ & AF LLVYLCI L & QQTLY DTAYL & M. mulatta & FYFLI I FL VQ & AF LI VYLF I L & QQP LY DAAYL \\
\hline C. lupus & FYFLI I FLVQ & VFLLVYLCI L & QQTLY DTAYL & C. lupus & FYFLI I FL VQ & AF L I VYLF I L & QQP LY DAAYL \\
\hline B. taurus & FYFLI I FLVQ & VF L VVYLCI L & QQT LY DTAYL & B. taurus & FYFLI I FLI Q & AF LI VYLF I L & QQP LY DAAYL \\
\hline M. musculus & FYFLI I FLVQ & TF L I VYLCI L & QQT LY DTAYL & M. musculus & FYFLI I FL VQ & AF LI VYLF I L & QQP LY DAAYL \\
\hline R. norvegicus & FYFLI I FLVQ & TF LI VYLCI L & QQTLY DTAYL & R. norvegicus & FYFLI I FL VQ & AF LI VYLF I L & QQP LY DAAYL \\
\hline G. gallus & FYFLI I F LVQ & VF LI VYLCI L & QQTLY DTAYL & G. gallus & FYFLI I FL VQ & AF LI VYLCI L & QQP LY DAAYL \\
\hline D. rerio & FYFLI I FLVQ & KS MNAYLI VY & QQTLY DTAYL & D. rerio & FYFLI I FL VQ & AF LI VYLCI L & QQP LY DAAYL \\
\hline X. tropicalis & FYFLI I FLVQ & AF LI VYLCI L & QQTLY DTAYL & $\mathrm{X}$. tropicalis & FYFLI I FL VQ & AF LI VYLCI L & QQP LY DAAYL \\
\hline
\end{tabular}

\begin{tabular}{|c|c|c|c|}
\hline TP8A1 & L127 & I344 & E981 \\
\hline H. sapiens & LYF LALLI LQ & YMVYTI FVVL & AQTAYEDWF I \\
\hline P. troglodytes & LYFLALLI LQ & YMVYTI FVVL & AQTAYEDWF I \\
\hline M. mulatta & LYFLVLLI LQ & YMVYTI FVVL & AQTAYEDWF I \\
\hline C. lupus & FYFLI LLI LQ & YMVYTI FVVL & AQTAYEDWF I \\
\hline B. taurus & FYF LVLLI LQ & YMVYTI FVVL & AQTAYE DWF I \\
\hline M. musculus & F YF LI LLI LQ & YMVYTI FVVL & AQTAYEDWF I \\
\hline R. norvegicus & FYF LI LLI LQ & YMVYTI FVVL & AQTAYE DUF I \\
\hline G. gallus & SYFLVLLI LQ & YMVYTI FVVL & AQTAYEDWF I \\
\hline D. rerio & LYF LCLLVLQ & YMVYTI FVVL & AQTAYEDWF I \\
\hline X. tropicalis & AYFLVLLI LQ & YMVYTI FVLL & AQTAYEDUF I \\
\hline
\end{tabular}

(a)

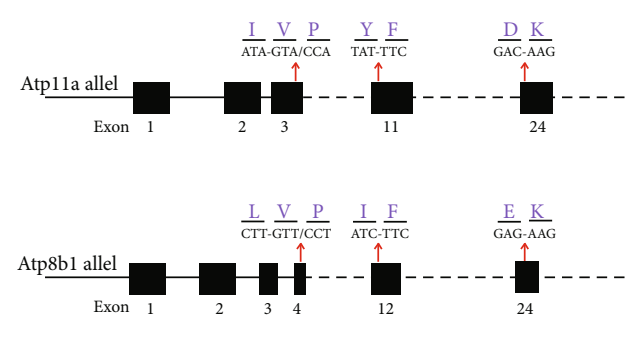

(b)

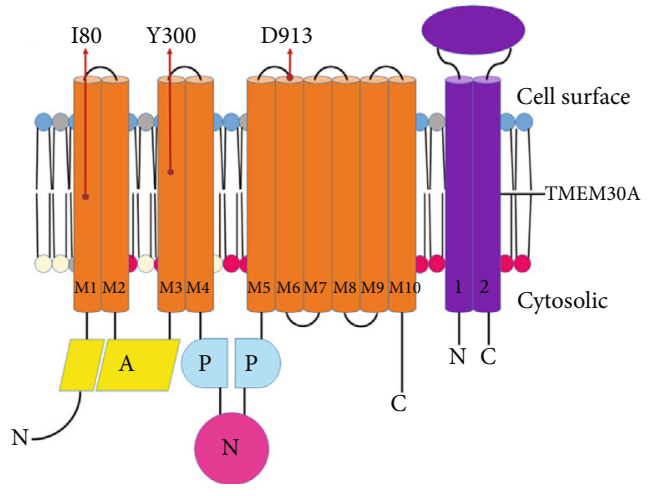

(c)

FIGURE 1: Location and conservation analysis of mutations affected residues. (a) ATP8B1 and ATP11A protein sequence alignment of amino acid sequences surrounding the identified mutations with its orthologues from $H$. sapiens to $X$. tropicalis. The affected amino acid residues are in red. Note that the amino acid sequences of ATP11A surrounding the affected amino acid residues are highly conserved among Homo sapiens, Pan troglodytes, Macaca mulatta, Canis lupus, Bos taurus, Mus musculus, Rattus norvegicus, Gallus gallus, Danio rerio, Xenopus tropicalis, Caenorhabditis elegans. (b) Schematic representation of exon-intron structure of Atp8b1 and Atp11a. (c) Topology of ATP11A with the location of the affected residues I80, Y300, and D913.

CA, USA). The Golgi apparatus was labeled with a rabbit anti-GM130 antibody ( $1: 1000$, BD Biosciences, Mississauga, $\mathrm{ON})$. The secondary antibodies used were Alexa 488 goat anti-rabbit IgG, Alexa 594 goat anti-mouse IgG (1:500, Invitrogen, USA). The images were captured under a Zeiss LSM 800 confocal scanning microscope.

2.5. Flippase Activity Assay. The following labeled phospholipid analogs were purchased from Avanti Polar Lipids: 16: 0-06: 0 NBD-PS [1-palmitoyl-2-[6-[(7-nitro-2-1,3-benzoxadiazol-4-yl)amino] hexanoyl]-sn-glycero-3-phospho-L-serine (ammonium salt)]. The translocation of NBD-lipid was determined as described with some modification [37]. 36-48 hours after transfection, COS7 cells were washed three times with PBS and treated with $0.25 \%$ trypsin. Cells were harvested by centrifugation and washed with HBSS (Waltham, MA, USA). Cells were incubated with $10 \mu \mathrm{M}$
18: 1 NBD-PS for 15 minutes at $25^{\circ} \mathrm{C}$. Subsequently, icecold HBSS supplemented with $2 \%$ bovine serum albumin (Sigma-Aldrich) was added to the cells for 10 minutes on ice to extract PS on the extracellular surface. This process was repeated 3 times. Subsequently, cells were centrifuged at $4^{\circ} \mathrm{C}$ for 5 minutes at $300 \mathrm{~g}$ and suspended in HBSS and analyzed with a flow cytometer (CytoFLEX, Beckman Coulter). The mean fluorescence intensity was calculated for each group. Dead cells were excluded from the analysis by blue fluorescence (DAPI positive). The NBD-lipid fluorescence intensity of living COS7 cells was plotted on a histogram to calculate the median fluorescence intensity. Lipid translocation activity was calculated as a ratio to WT control samples.

2.6. Statistical Analysis. Statistical analysis was performed by one-way analysis of variance or student's $t$-test using GraphPad Prism 6 software. The differences were considered 


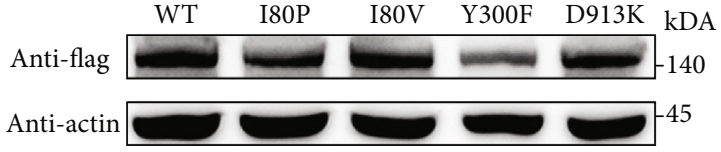

(a)

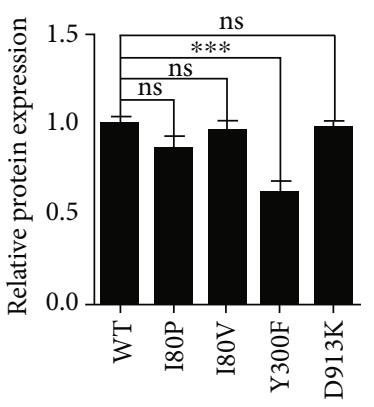

(b)

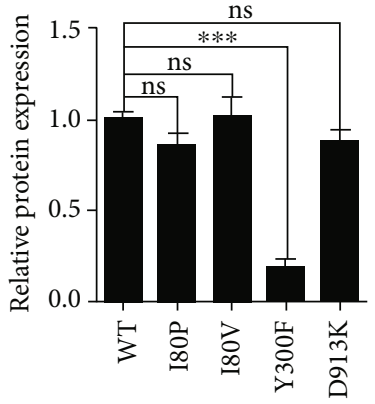

(c)
FIGURE 2: Expression analysis of WT and mutant ATP11A proteins in HEK 293T cells when co-expressed with TMEM30A. (a) Western blot analysis of ATP11A-WT, ATP11A-I80P, ATP11A-I80V, ATP11A-Y300F, and ATP11A-D913K in HEK 293T cells using anti-Flag antibody. $\beta$-Actin was used as the loading control. Uncropped gel pictures were shown in Figure S1. (b) Quantification of WT and mutant ATP11A proteins revealed that the expression level of ATP11A-Y300F was reduced. ATP11A-WT was used as control. $N=3 .{ }^{* *} p<0.01$. The data represented mean \pm SEM. (c) Quantitative real-time PCR analysis of WT and mutant ATP11A mRNA revealed that the transcriptional level of ATP11A-Y300F mutant was reduced. ATP11A-WT was used as control. $N=5$. ${ }^{* *} p<0.01$. ns: no statistical significance. The data represented mean \pm SEM. A representative result of three independent experiments was shown for $\mathrm{a}-\mathrm{c}$.

statistically significant at $p$ values $<0.05$. The quantitative data are presented as the mean \pm SEM as indicated in the figure legends. All experiments were performed in triplicate and repeated at least twice.

\section{Results}

3.1. Conservative Analysis of ATP11A Residues. Previous studies $[33,34]$ reported that amino residues affected by ATP8B1 mutations found in patients, L127P, I344F, and E897K, are highly conserved among 10 different species (Figures 1(a) and 1(b)). Comparative amino acid sequence alignment of ATP11A proteins across different species revealed that the corresponding I80, Y300, and D913 residues were also highly conserved (Figure 1). ATP11A contains ten transmembrane helices and $\mathrm{A}, \mathrm{P}$, and $\mathrm{N}$ domains and the $\mathrm{C}$-terminal regulatory domain $[38,39]$ (Figure 1(c)). I80 and Y300 are located in the transmembrane region, while D913 is located at the exoplasmic loop between transmembrane helices 5 and 6 (Figure 1(c)). These data indicated that the corresponding residues of ATP11A might be critical for its expression and function.

3.2. The Y300F Variant of ATP11A Impairs Its Expression Level. To evaluate the impact of the I80P, I80V, Y300F, and D913K variants on ATP11A expression, we introduced I80P, I80V, Y300F, and D913K variants unto ATP11A and assessed their expression levels. We transfected plasmids of ATP11AWT, ATP11A-I80P, ATP11A-I80V, ATP11A-Y300F, and ATP11A-D913K, which carried a C-terminal $3 \times$ Flag tag, into HEK 293T cells. Cell lysates were separated by SDS-PAGE and subsequently subjected to immunoblotting analysis. The molecular weight of human ATP11A calculated from its primary structures is $129.7 \mathrm{kDa}$ (UniProtKB-P98196), whereas ATP11A protein was detected by immunoblotting with antibodies against Flag at approximately $142 \mathrm{kDa}$ (Figure 2(a)). Considering the added molecular weight by $3 \times$ Flag tag, the calculated molecular weight is $133 \mathrm{kDa}$. The detected $142 \mathrm{kDa}$ band is bigger than the calculated molecular weight, probably due to posttranslational modification. The immunoblotting data revealed that the expression level of ATP11AY300F in HEK 293T cells was reduced $40 \%$ compared to ATP11A-WT (Figure 2(b)), while that of other ATP11A variants seems unchanged. RT-PCR analysis showed a reduced mRNA level for the ATP11A-Y300F mutant (Figure 2(c)). Therefore, Y300F mutation affected the transcriptional level and then caused the reduced content of protein.

3.3. The Y300F and D913K Variants Impairs the Proper Subcellular Localization of ATP11A in the Presence of TMEM30A. In order to detect the effect of mutations on the cellular localization of ATP11A, we first investigated the intracellular localization of variants by immunocytochemistry in the context of transfection of ATP11A without TMEM30A in COS7 cells. ATP11A-WT was largely localized to the endoplasmic reticulum (ER), which was manifested by the specific ER marker Calnexin, and its mutants presented similar localization patterns (Figure 3), suggesting little impact of these variants in ATP11A subcellular localization without overexpression of TMEM30A.

Previous studies have indicated that TMEM30A plays a key role in the correct localization of PS flippases [40, 41]. We further investigated the effect of TMEM30A coexpression on the subcellular localization of ATP11A by confocal microscopy. As mentioned above, when ATP11A and its mutants were single transfected into COS7 cells, they were largely localized to ER (Figure 3). However, when ATP11A was cotransfected with TMEM30A into COS7, a large extent of ATP11A exit from ER to Golgi and on the plasma membrane. This indicated that TMEM30A might help the correct localization of ATP11A (Figure 4).

We further investigated the impact of these variants on cellular localization in the presence of TMEM30A. Notably, ATP11A-I80P and ATP11A-I80V were mostly localized to the Golgi apparatus and plasma membrane, while ATP11AY300F and ATP11A-D913K still resided at ER improperly (Figure 4(a)). Statistical data manifested that the colocalization ratio of ATP11A-WT, ATP11A-I80P, and ATP11AI80V with Golgi was about 60-70\%, while that of ATP11AY300F and ATP11A-D913K with Golgi was only 20-30\% (Figure 4(b)). These results demonstrated that ATP11A-I80P and ATP11A-I80V did not affect the correct sorting and targeting of mutant ATP11A proteins, whereas ATP11A-Y300F and ATP11A-D913K might obstruct the interaction with TMEM30A, resulting in the failure of positioning to Golgi apparatus with the help of TMEM30A. 

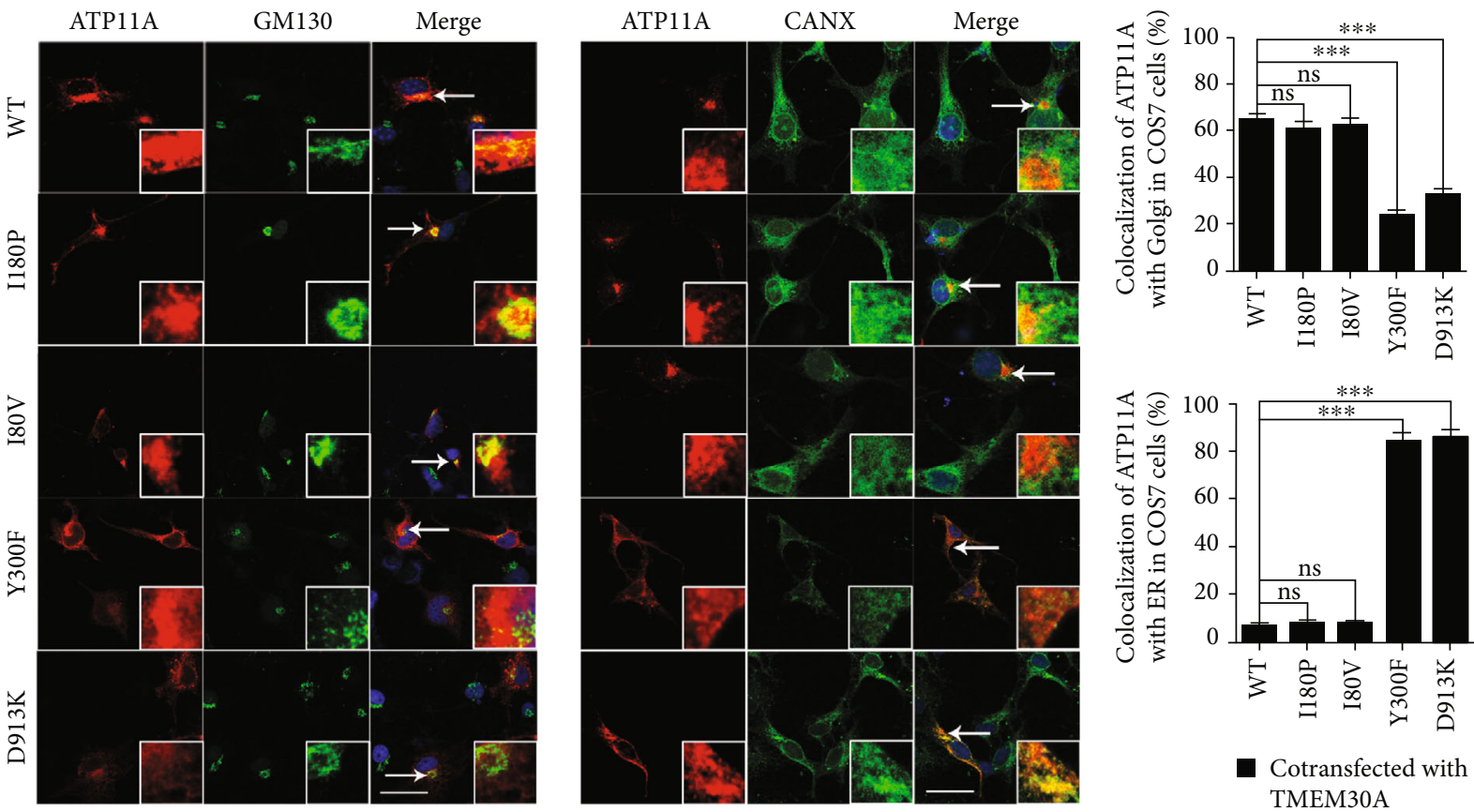

(a)

- Cotransfected with TMEM30A

(b)

FIgURE 3: Subcellular localization of ATP11A and three mutants in COS7 cells. (a) COS7 cells were transiently transfected with 3X-FLAg tagged WT or mutant ATP11A plasmids. Cells were double stained for FLAG and organelle marker GM130 or Calnexin (CANX). Nuclei were counter-stained with DAPI (blue). (b) Percentage of colocalization of ATP11A with GM130-marked Golgi (upper panel) and CANX-marked ER (lower panel) in COS7 cells. $N=6$. ns: no statistical difference. Data are presented as the mean \pm SEM. Scale bars: $25 \mu \mathrm{m}$.

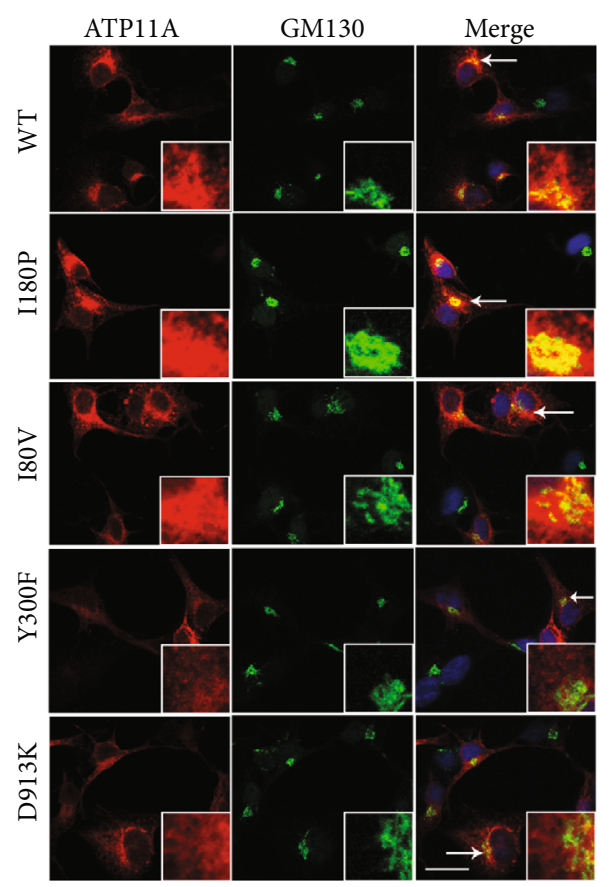

(a)

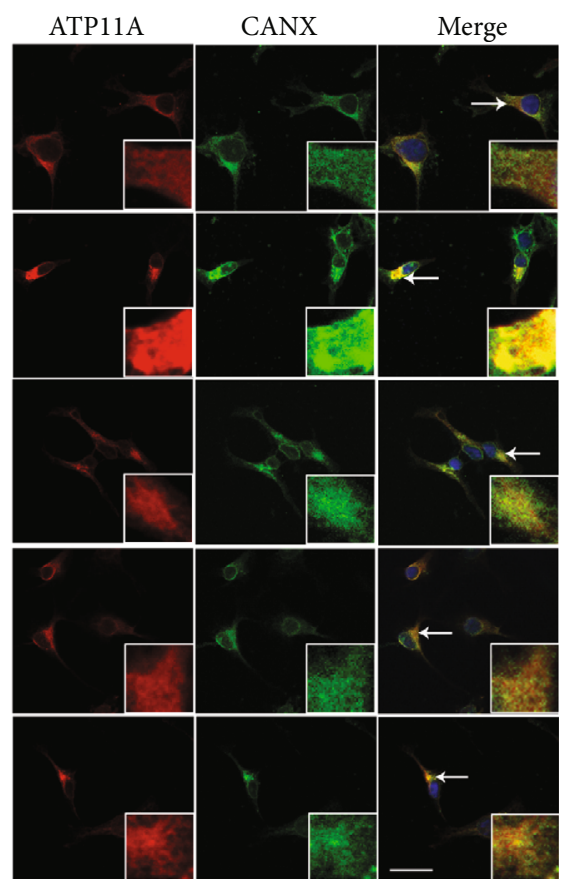

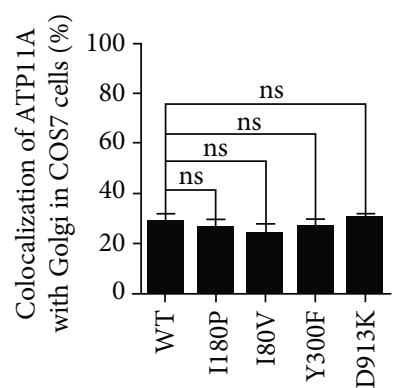

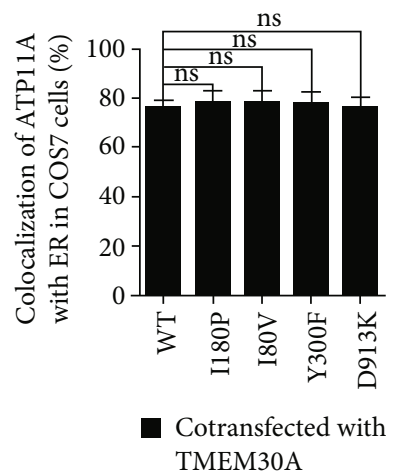

(b)

FIGURE 4: Subcellular localization of ATP11A mutants when cotransfected with TMEM30A in COS7 cells. (a) COS7 cells were transiently transfected with FLAG tagged ATP11A and HA-tagged TMEM30A plasmids. Cells were double stained for FLAG and organelle marker GM130 or Calnexin (CANX). Nuclei were counterstained with DAPI (blue). Scale bars: $25 \mu \mathrm{m}$. (b) Percentage of colocalization of cotransfected ATP11A and TMEM30A with GM130-marked Golgi (upper panel) and CANX-marked ER (lower panel) in COS7 cells. $N$ $=6 .{ }^{* *} p<0.01 ;{ }^{* * *} p<0.001$. Data are presented as the mean \pm SEM. Scale bars: $25 \mu \mathrm{m}$. 


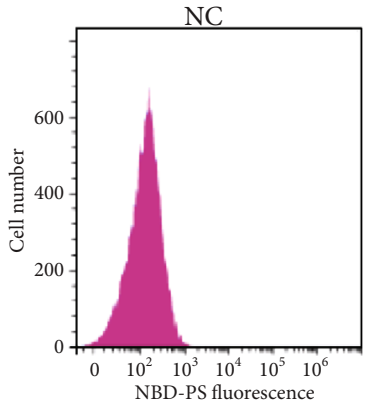

(a)

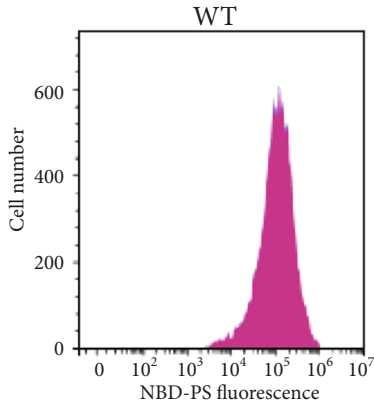

(b)

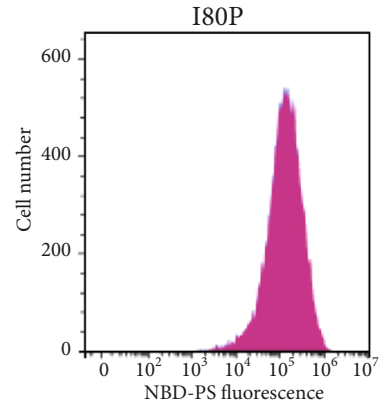

(c)

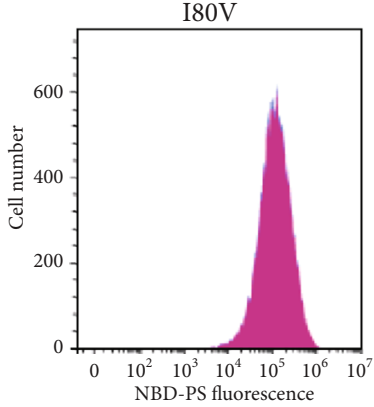

(d)

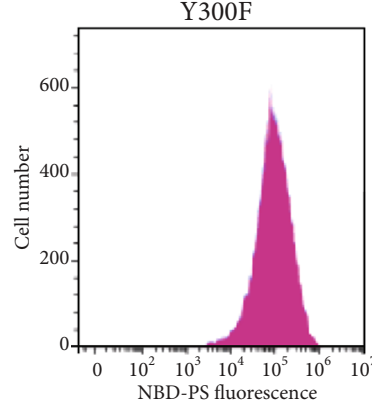

(e)

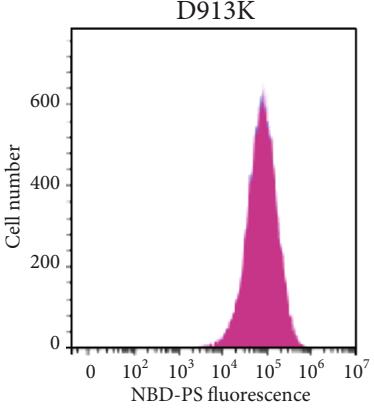

(f)

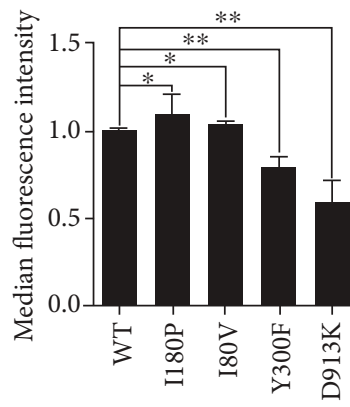

(g)

FIGURE 5: PS flippase activity assay of ATP11A mutants. (a-g) NBD-PS labeling assays revealed a reduced amount of PS transported into the cells for ATP11A Y300F and D913K mutants. Flow cytometry histograms of NBD-PS labeling assays were shown in a-f. The $x$-axis represented the NBD-PS fluorescence intensity of intact living cells. The $y$-axis showed numbers of NBD-PS-labeled cells. PcDNA3.1-C3xflag empty plasmid was transfected as a negative control (NC) (a). PS flippase activity measured by the mean fluorescence intensity of ATP11A mutant transfected cells (f). ATP11A-WT was used as control and its activity was set as $1.0 . N=5 .^{* *} p<0.01$. The data represented mean \pm SEM.

\subsection{The Y300F and D913K Variants Resulted in Reduced PS} Internalization in Plasma Membrane. To investigate whether these mutations affect the phospholipid flippase activity, fluorescence-labeled PS analog (NBD-PS) was added into COS7 cells, which were transfected with the WT or variants plasmids of ATP11A. After incubation with NBD-PS for 15 minutes at room temperature, the cells were treated with $2 \%$ BSA to remove NBD-PS at the outer layer, and the fluorescence intensity of the inner layer was measured by flow cytometry (Figures 5(a)-5(e)). Compared to ATP11AWT transfected cells, median NBD-PS fluorescence intensity (MFI) in ATP11A-Y300F and ATP11A-D913K transfected cells was decreased to $70 \%$ and $60 \%$ of the WT controls under the similar experimental conditions, suggesting reduced PS internalization in the plasma membrane (Figure 5(f)). These data demonstrated that variants of Y300F and D913K resulted in reduced PS internalization in the plasma membrane, which might be caused by a reduction in the amount of ATP11A or a decrease in the activity of ATP11A on the plasma membrane.

\section{Discussion}

Eukaryotic P4-ATPases plays important roles in various cellular processes. Atp8a2 was reported to play a role in promoting neurite outgrowth in neuronal PC12 cells and rat hippocampal neurons [42]. Studies in mouse models have contributed to our understanding of the physiological functions of mammalian P4ATPases: Atp8a1-deficient mice exhibit delayed hippocampus-dependent learning, Atp8a2mutant mice display neurological abnormalities, and Atp11c-deficient mice show arrested B-cell development [22]. However, little is known about the in vivo function of ATP11A. In order to get a deeper understanding of ATP11A, we selected three mutation sites L127, I344, and E981 screened by ATP8B1, a member of the homologous family, as a reference. An ATP8B1 homozygous mutation ATP8B1L127V causes intrahepatic cholestasis in two Omani siblings [35]. Mutation of ATP8B1-L127P has been reported to be related to PFIC1, but this mutant did not cause any change in the expression level and subcellular localization of ATP8B1 [33, 43]. ATP8B1-I344F detected in European families can cause BRIC1 [33]. A case of PFIC1 with mutation of ATP8B1-E981K in the Japanese family was reported [34].

According to the amino acid sequence homology alignment, the equivalent positions of I80P, I80V, Y300F, and D913K were determined in ATP11A. Mutant expression plasmids were constructed in vitro, and its expression level, localization, and activity of flippase in the cell were explored. We found that the expression of a variant of Y300F was reduced by $40 \%$ by immunoblotting (Figure 2), indicating that mutation at this site may lead to degradation of ATP11A 


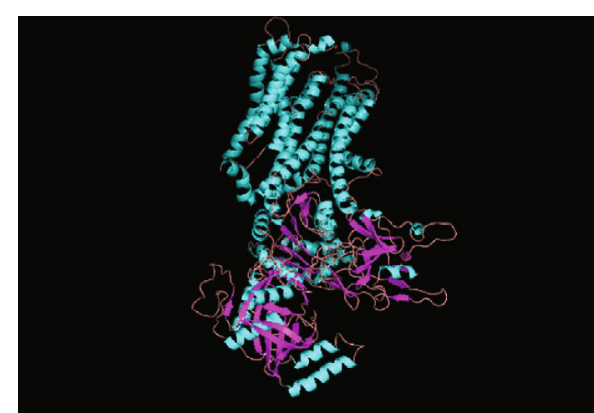

(a)

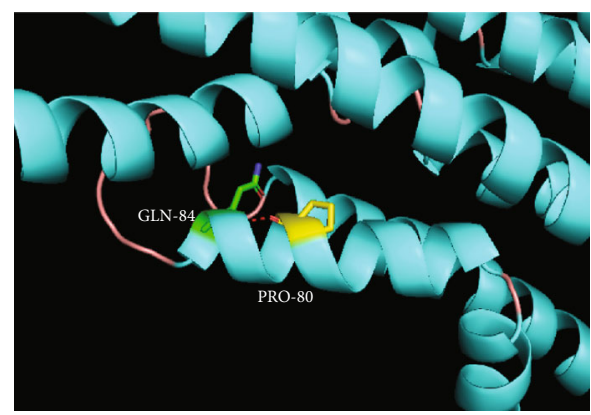

(c)

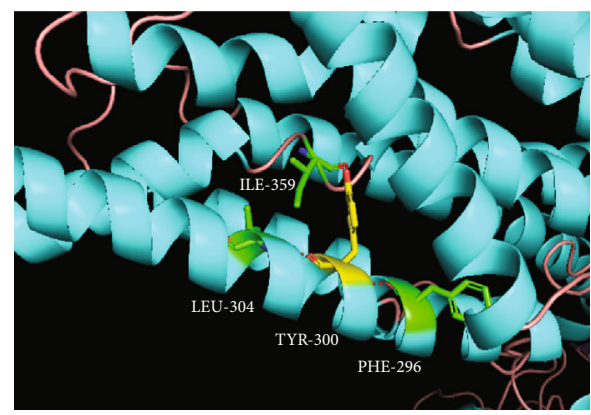

(e)

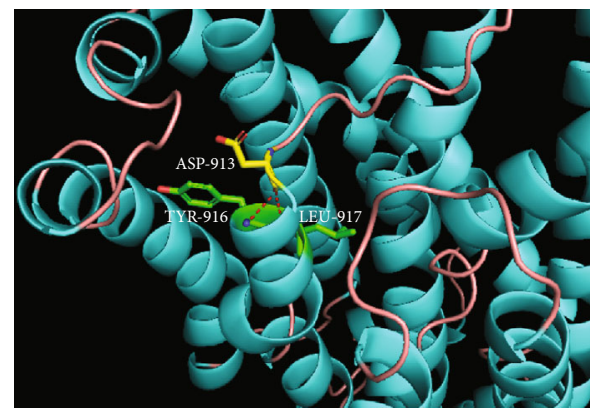

(g)

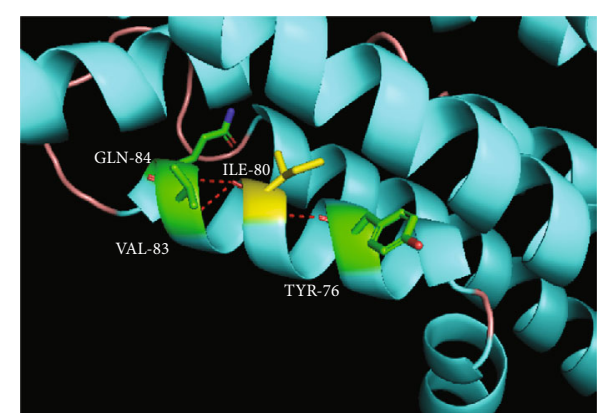

(b)

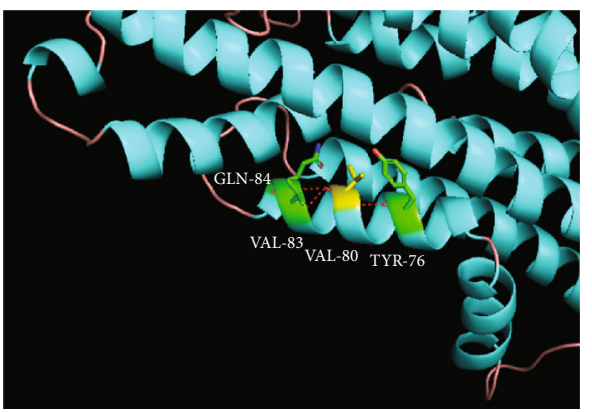

(d)

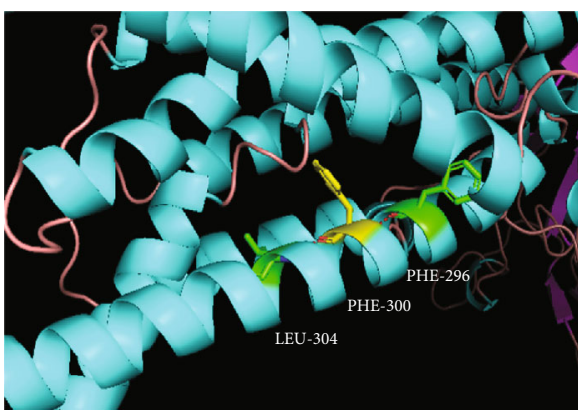

(f)

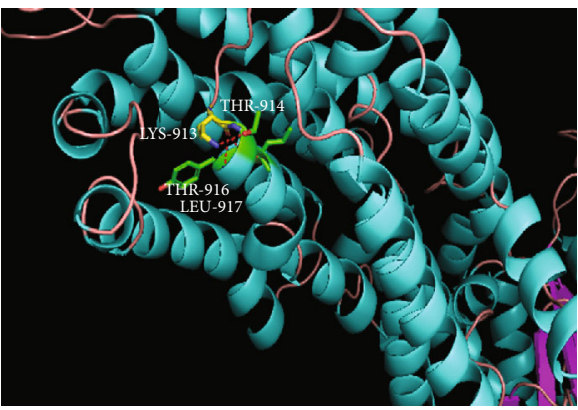

(h)

Figure 6: Models of ATP11A mutants. (a) A homology model of ATP11A was constructed based on sequence alignment with other P-type ATPases and available structural data. The transmembrane helices, loop, and sheet are blue, pink, and purple, respectively. (b-h) Target amino acids are shown in yellow, amino acids linked to the target amino acid are shown in green, and hydrogen bonds are shown in red. (b-d) I80 residue is linked to Y76, V83, and Q84, and V80 residue has the same connection. However, P80 residue is only linked to Q84. (e, f) Y300 residue is linked to F296, L304, and nearby I359 positioned on the M3-M4 loop. Nevertheless, F300 residue cannot be connected to I359. (g, h) D913 residue, located at the M5-M6 loop, is linked to Y916 and L917. While the direction of K913 residue changed, and one more T914 was connected.

protein. This mutation site is located in the third transmembrane domain (M3) of ATP11A protein (Figure 1(c)) and is highly conserved in all 10 species sequences (Figure 1(a)). In the crystal model, phenylalanine cannot be properly linked to isoleucine at position 359 on the M3-M4 loop (Figure 6). This indicates that mutations affecting this residue may lead to protein misfolding and eventually degradation by the proteasome. 
In addition, our immunocytochemistry experiments demonstrated that variants of $\mathrm{Y} 300 \mathrm{~F}$ and D913K could not be correctly located in the Golgi apparatus and plasma membrane when cotransfected with TMEM30A (Figure 4(a)). Residues of Y300 and D913 are located at M3 and M5-M6 loop of ATP11A protein, respectively (Figure $1(\mathrm{c})$ ). In the crystal model, the variant Y300F causes the M3 and M3M4 loop to be incorrectly connected (Figure 6), and variant D913K causes an error in the connection of M6 to M5-M6 loop (Figure 6). M3-M4 and M5-M6 loops are on the cell surface (Figure 1(c)). It has been reported that in the extracellular region, the CDC50A ectodomain covers all of the extracellular loops of ATP8A1, except for the M1-2 loop, interacting in an electrostatic complementary manner: the extracellular loops of ATP8A1 bear negative charges, whereas CDC50A bears positive charges [38]. Therefore, we speculated that these two mutations interfere with the normal binding of ATP11A and TMEM30A and eventually cause ATP11A to be incorrectly located. Variants I80P and I80V only altered the connection of adjacent amino acids, but did not change the connection between the M1 and other loops (Figures 6(c) and 6(d)). This also explains that these two variants have little effect on the ATP11A protein.

In summary, our data indicated that the Y300F mutation of ATP11A could cause a decrease in protein expression, and variants of Y300F and D913K affected the subcellular localization of ATP11A. These data indicate that the Y300 and D913 residues are important in the normal function of the ATP11A protein. Further investigation into the in vivo function of ATP11A is warranted.

\section{Data Availability}

All data are included in the manuscript.

\section{Disclosure}

The funders had no role in study design, data collection, and analysis or preparation of the manuscript.

\section{Conflicts of Interest}

The authors declare that they have no conflicts of interest.

\section{Authors' Contributions}

Kuanxiang Sun and Wanli Tian are co-first authors and contributed equally to this work.

\section{Acknowledgments}

This study was supported by grants from the Department of Science and Technology of Sichuan Province (http://kjt.sc.gov .cn/, 2020JDZH0027, 2019ZYD002, 2018YSZH0020 and 2018JZ0019).

\section{Supplementary Materials}

Figure S1: the uncropped gel images for Figure 2. (Supplementary Materials)

\section{References}

[1] R. L. Lopez-Marques, L. Theorin, M. G. Palmgren, and T. G. Pomorski, "P4-ATPases: lipid flippases in cell membranes," Pflügers Archiv, vol. 466, no. 7, pp. 1227-1240, 2014.

[2] M. Seigneuret and P. F. Devaux, "ATP-dependent asymmetric distribution of spin-labeled phospholipids in the erythrocyte membrane: relation to shape changes," Proceedings of the National Academy of Sciences of the United States of America, vol. 81, no. 12, pp. 3751-3755, 1984.

[3] T. R. Graham, "Flippases and vesicle-mediated protein transport," Trends in Cell Biology, vol. 14, no. 12, pp. 670-677, 2004.

[4] R. Panatala, H. Hennrich, and J. C. M. Holthuis, "Inner workings and biological impact of phospholipid flippases," Journal of Cell Science, vol. 128, no. 11, pp. 2021-2032, 2015.

[5] M. S. Halleck, J. F. Lawler, S. Blackshaw et al., "Differential expression of putative transbilayer amphipath transporters," Physiological Genomics, vol. 1, no. 3, pp. 139-150, 1999.

[6] C. C. Paulusma and R. P. Oude Elferink, "The type 4 subfamily of P-type ATPases, putative aminophospholipid translocases with a role in human disease," Biochimica et Biophysica Acta (BBA) - Molecular Basis of Disease, vol. 1741, no. 1-2, pp. 11-24, 2005.

[7] K. Segawa, S. Kurata, Y. Yanagihashi, T. R. Brummelkamp, F. Matsuda, and S. Nagata, "Caspase-mediated cleavage of phospholipid flippase for apoptotic phosphatidylserine exposure," Science, vol. 344, no. 6188, pp. 1164-1168, 2014.

[8] C.-Y. Chen, M. F. Ingram, P. H. Rosal, and T. R. Graham, "Role for Drs2p, a P-type ATPase and potential aminophospholipid translocase, in yeast late Golgi function," Journal of Cell Biology, vol. 147, no. 6, pp. 1223-1236, 1999.

[9] J. A. Coleman, F. Quazi, and R. S. Molday, "Mammalian P4ATPases and ABC transporters and their role in phospholipid transport," Biochimica et Biophysica Acta (BBA) - Molecular and Cell Biology of Lipids, vol. 1831, no. 3, pp. 555-574, 2013.

[10] D. E. Folmer, R. P. J. O. Elferink, and C. C. Paulusma, "P4 ATPases - Lipid flippases and their role in disease," Biochimica et Biophysica Acta (BBA) - Molecular and Cell Biology of Lipids, vol. 1791, no. 7, pp. 628-635, 2009.

[11] E. Y. Gong, E. Park, H. J. Lee, and K. Lee, "Expression of Atp8b3 in murine testis and its characterization as a testis specific P-type ATPase," Reproduction, vol. 137, no. 2, pp. 345$351,2009$.

[12] H. M. Hankins, R. D. Baldridge, P. Xu, and T. R. Graham, "Role of flippases, scramblases and transfer proteins in phosphatidylserine subcellular distribution," Traffic, vol. 16, no. 1, pp. 35-47, 2015.

[13] T. Naito, H. Takatsu, R. Miyano, N. Takada, K. Nakayama, and H. W. Shin, "Phospholipid flippase ATP10A translocates phosphatidylcholine and is involved in plasma membrane dynamics," Journal of Biological Chemistry, vol. 290, no. 24, pp. 15004-15017, 2015.

[14] S. Nagata, et al.J. Suzuki, K. Segawa, and T. Fujii, "Exposure of phosphatidylserine on the cell surface," Cell Death \& Differentiation, vol. 23, no. 6, pp. 952-961, 2016.

[15] T. T. Sebastian, R. D. Baldridge, P. Xu, and T. R. Graham, "Phospholipid flippases: building asymmetric membranes and transport vesicles," Biochimica et Biophysica Acta (BBA) - Molecular and Cell Biology of Lipids, vol. 1821, no. 8, pp. 1068-1077, 2012. 
[16] L. Wang, C. Beserra, and D. L. Garbers, "A novel aminophospholipid transporter exclusively expressed in spermatozoa is required for membrane lipid asymmetry and normal fertilization," Developmental Biology, vol. 267, no. 1, pp. 203-215, 2004.

[17] X. Tang, M. S. Halleck, R. A. Schlegel, and P. Williamson, “A subfamily of P-type ATPases with aminophospholipid transporting activity," Science, vol. 272, no. 5267, pp. 1495-1497, 1996.

[18] H. Takatsu, M. Takayama, T. Naito et al., "Phospholipid flippase ATP11C is endocytosed and downregulated following Ca2+-mediated protein kinase C activation," Nature Communications, vol. 8, no. 1, p. 1423, 2017.

[19] K. Segawa, S. Kurata, and S. Nagata, "Human type IV P-type ATPases that work as plasma membrane phospholipid flippases and their regulation by caspase and calcium," Journal of Biological Chemistry, vol. 291, no. 2, pp. 762-772, 2016.

[20] V. van der Mark, R. Elferink, and C. Paulusma, "P4 ATPases: flippases in health and disease," International Journal of Molecular Sciences, vol. 14, no. 4, pp. 7897-7922, 2013.

[21] S. Bryde, H. Hennrich, P. M. Verhulst, P. F. Devaux, G. Lenoir, and J. C. M. Holthuis, "CDC50 proteins are critical components of the human class- $1 \mathrm{P}_{4}$-ATPase transport machinery," Journal of Biological Chemistry, vol. 285, pp. 40562-40572, 2010.

[22] J. P. Andersen, A. L. Vestergaard, S. A. Mikkelsen, L. S. Mogensen, M. Chalat, and R. S. Molday, "P4-ATPases as phospholipid flippases-structure, function, and enigmas," Frontiers in Physiology, vol. 7, p. 275, 2016.

[23] L. N. Bull, M. J. T. van Eijk, L. Pawlikowska et al., "A gene encoding a P-type ATPase mutated in two forms of hereditary cholestasis," Nature Genetics, vol. 18, no. 3, pp. 219-224, 1998.

[24] J. M. Stapelbroek, T. A. Peters, D. H. A. van Beurden et al., "ATP8B1 is essential for maintaining normal hearing," Proceedings of the National Academy of Sciences of the United States of America, vol. 106, no. 24, pp. 9709-9714, 2009.

[25] O. E. Onat, S. Gulsuner, K. Bilguvar et al., "Missense mutation in the ATPase, aminophospholipid transporter protein ATP8A2 is associated with cerebellar atrophy and quadrupedal locomotion," European Journal of Human Genetics, vol. 21, no. 3, pp. 281-285, 2013.

[26] J. A. Coleman, X. Zhu, H. R. Djajadi et al., "Phospholipid flippase ATP8A2 is required for normal visual and auditory function and photoreceptor and spiral ganglion cell survival," Journal of Cell Science, vol. 127, Part 5, pp. 1138-1149, 2014.

[27] O. M. Siggs, C. N. Arnold, C. Huber et al., "The P4-type ATPase ATP11C is essential for B lymphopoiesis in adult bone marrow," Nature Immunology, vol. 12, no. 5, pp. 434-440, 2011.

[28] M. Yabas, C. E. Teh, S. Frankenreiter et al., "ATP11C is critical for the internalization of phosphatidylserine and differentiation of B lymphocytes," Nature Immunology, vol. 12, no. 5, pp. 441-449, 2011.

[29] O. M. Siggs, B. Schnabl, B. Webb, and B. Beutler, "X-linked cholestasis in mouse due to mutations of the P4-ATPase ATP11C," Proceedings of the National Academy of Sciences of the United States of America, vol. 108, no. 19, pp. 7890-7895, 2011.

[30] M. Yabas, et al.L. A. Coupland, D. Cromer et al., "Mice deficient in the putative phospholipid flippase ATP11C exhibit altered erythrocyte shape, anemia, and reduced erythrocyte life span," Journal of Biological Chemistry, vol. 289, no. 28, pp. 19531-19537, 2014.

[31] H. Takatsu, G. Tanaka, K. Segawa et al., "Phospholipid flippase activities and substrate specificities of human type IV P-type ATPases localized to the plasma membrane," Journal of Biological Chemistry, vol. 289, no. 48, pp. 33543-33556, 2014.

[32] M. Tsuchiya, Y. Hara, M. Okuda et al., "Cell surface flip-flop of phosphatidylserine is critical for PIEZO1-mediated myotube formation," Nature Communications, vol. 9, no. 1, p. 2049, 2018.

[33] L. W. J. Klomp, J. C. Vargas, S. W. C. van Mil et al., "Characterization of mutations inATP8B1associated with hereditary cholestasis," Hepatology, vol. 40, no. 1, pp. 27-38, 2004.

[34] C. Numakura, D. Abukawa, T. Kimura, S. Tanabe, and K. Hayasaka, "A case of progressive familial intrahepatic cholestasis type 1 with compound heterozygous mutations of ATP8B1," Pediatrics International, vol. 53, no. 1, pp. 107110, 2011.

[35] H. Narchi, S. Alhefeiti, F. Althabahi, J. Hertecant, A. S. Knisely, and A. K. Souid, "Intrahepatic cholestasis in two omani siblings associated with a novel homozygous ATP8B1 mutation, c.379C>G (p.L127V)," Saudi Journal of Gastroenterology, vol. 23, no. 5, pp. 303-305, 2017.

[36] R. H. Gantzel, L. S. Mogensen, S. A. Mikkelsen et al., "Disease mutations reveal residues critical to the interaction of P4ATPases with lipid substrates," Scientific Reports, vol. 7, no. 1, p. 10418, 2017.

[37] X. Zhu, R. T. Libby, W. N. de Vries et al., "Mutations in a Ptype ATPase gene cause axonal degeneration," PLoS Genetics, vol. 8, no. 8, article e1002853, 2012.

[38] M. Hiraizumi, K. Yamashita, T. Nishizawa, and O. Nureki, "Cryo-EM structures capture the transport cycle of the P4ATPase flippase," Science, vol. 365, no. 6458, pp. 1149-1155, 2019.

[39] M. Timcenko, J. A. Lyons, D. Januliene et al., "Structure and autoregulation of a P4-ATPase lipid flippase," Nature, vol. 571, no. 7765, pp. 366-370, 2019.

[40] Z. Schultzhaus, G. A. Cunningham, R. R. Mouriño-Pérez, and B. D. Shaw, "The phospholipid flippase DnfD localizes to late Golgi and is involved in asexual differentiation in Aspergillus nidulans," Mycologia, vol. 111, no. 1, pp. 13-25, 2019.

[41] L. R. Poulsen, R. L. López-Marqués, S. C. McDowell et al., "The Arabidopsis P4-ATPase ALA3 localizes to the golgi and requires a beta-subunit to function in lipid translocation and secretory vesicle formation," The Plant Cell, vol. 20, no. 3, pp. 658-676, 2008.

[42] Q. Xu, G.-Y. Yang, N. Liu et al., "P4-ATPase ATP8A2 acts in synergy with CDC50A to enhance neurite outgrowth," Febs Letters, vol. 586, no. 13, pp. 1803-1812, 2012.

[43] L. M. van der Velden, J. M. Stapelbroek, E. Krieger et al., "Folding defects in P-type ATP 8B1 associated with hereditary cholestasis are ameliorated by 4-phenylbutyrate," Hepatology, vol. 51, no. 1, pp. 286-296, 2010. 